\title{
THE MAXIMUM PRINCIPLE FOR VECTOR FIELDS
}

\author{
FRANK H. BEATROUS, THOMAS J. BIESKE, AND JUAN J. MANFREDI
}

\begin{abstract}
We present an extension of Jensen's uniqueness theorem for viscosity solutions of second order partial differential equations to the case of equations generated by vector fields.
\end{abstract}

\section{INTRODUCTION}

In a celebrated theorem R. Jensen $[\mathrm{J}]$ established the uniqueness of viscosity solutions of fully non-linear second order partial differential equations in $\mathbb{R}^{n}$. These equations are of the form

$$
F\left(x, u(x), \nabla u(x), D^{2} u(x)\right)=0,
$$

where $x$ is in some domain $\Omega \subset \mathbb{R}^{n}$, the function $u: \Omega \mapsto \mathbb{R}$ is real valued, the gradient $\nabla u$ is the vector $\left(\partial_{x_{1}} u, \partial_{x_{2}} u, \ldots, \partial_{x_{n}} u\right)$, and the second derivatives $D^{2} u$ is the $n \times n$ symmetric matrix with entries $\partial_{x_{i} x_{j}}^{2} u$. Jensen's theorem was later crafted in the language of jets and extended in [CIL]. In this latter reference, Jensen's theorem follows from the Maximum Principle for Semi-continuous Functions.

In this article we present and extension of the Crandall-Ishii-Lions maximum principle for semi-continuous functions and investigate the analogue of Jensen's theorem when the vector fields $\left\{\partial_{1}, \partial_{x_{2}}, \ldots, \partial_{x_{n}}\right\}$ are replaced by an arbitrary collection of vector fields or frame

$$
\mathfrak{X}=\left\{X_{1}, X_{2}, \ldots, X_{m}\right\} .
$$

The natural gradient is the vector

$$
D_{\mathfrak{X}} u=\left(X_{1}(u), X_{2}(u), \ldots, X_{m}(u)\right)
$$

and the natural second derivative is the $m \times m$ not necessarily symmetric matrix with entries $X_{i}\left(X_{j}(u)\right)$. Two important examples are:

(i) when $m=n$ and the frame $\mathfrak{X}$ is the orthonormal frame determined by a Riemannian metric, and

(ii) when $m<n$ and the frame $\mathfrak{X}$ satisfies the Hörmander condition

$$
\operatorname{dim}\left(\text { Lie Algebra } \operatorname{span}\left\{X_{1}, X_{2}, \ldots, X_{m}\right\}(x)\right)=n .
$$

Our main result, see Theorem 1 below, extends the maximum principle for semi-continuous functions to the case (i). In case (ii) an extension of Jensen's theorem has been recently found by Wang $[\mathrm{W}]$ when the frame $\mathfrak{X}$ is

Date: March 25, 2004.

Third author partially supported by NSF award DMS-0100107. 
the horizontal subspace of the graded Lie algebra of a Carnot group. To the best of our knowledge the general case of Hörmander vector fields without group structure remains open.

\section{TAYlor Formula for Vector Fields}

Consider a frame $\mathfrak{X}=\left\{X_{1}, X_{2}, \ldots, X_{n}\right\}$ in $\mathbb{R}^{n}$ consisting of $n$ linearly independent smooth vector fields. Write

$$
X_{i}(x)=\sum_{j=1}^{n} a_{i j}(x) \frac{\partial}{\partial x_{j}}
$$

for smooth functions $a_{i j}(x)$. Denote by $\mathbb{A}(x)$ the matrix whose $(i, j)$-entry is $a_{i j}(x)$. We always assume that $\operatorname{det}(\mathbb{A}(x)) \neq 0$ in $\mathbb{R}^{n}$.

We first write down an appropriate Taylor theorem adapted to the frame $\mathfrak{X}$. For this, we will use exponential coordinates as done in [NSW]. Fix a point $p \in \mathbb{R}^{n}$ and let $t=\left(t_{1}, t_{2}, \ldots, t_{n}\right)$ denote a vector close to zero. We define the exponential based at $p$ of $t$, denoted by $\Theta_{p}(t)$, as follows: Let $\gamma$ be the unique solution to the system of ordinary differential equations

$$
\gamma^{\prime}(s)=\sum_{i=1}^{n} t_{i} X_{i}(\gamma(s))
$$

satisfying the initial condition $\gamma(0)=p$. We set $\Theta_{p}(t)=\gamma(1)$ and note this is defined in a neighborhood of zero.

Recall that the natural gradient of a function $u$ relative to the frame $\mathfrak{X}$ is $D_{\mathfrak{X}} u=\left(X_{1}(u), X_{2}(u), \ldots, X_{n}(u)\right)$ and that the second derivative matrix $D_{\mathfrak{X}}^{2} u$ is the $n \times n$ matrix with entries $X_{i}\left(X_{j}(u)\right)$. We shall be interested in the quadratic form determined by this matrix, which is the same as the quadratic form determined by the symmetrized second derivative

$$
\left(D_{\mathfrak{X}}^{2} u\right)^{*}=\frac{1}{2}\left(D_{\mathfrak{X}}^{2} u+\left(D_{\mathfrak{X}}^{2} u\right)^{t}\right) .
$$

Applying the one-dimensional Taylor's formula to $u(\gamma(s))$ we get

Lemma 1. ([NSW]) Let $u$ be a smooth function in a neighborhood of $p$. We have:

$$
u\left(\Theta_{p}(t)\right)=u(p)+\left\langle D_{\mathfrak{X}} u(p), t\right\rangle+\frac{1}{2}\left\langle\left(D_{\mathfrak{X}}^{2} u(p)\right)^{*} t, t\right\rangle+o\left(|t|^{2}\right)
$$

as $t \rightarrow 0$.

For this lemma to hold we do not need to have $n$-linearly independent vector fields. The vector fields do not have to be independent and their number does not have to match the dimension. See [NSW].

Applying lemma (1) to the coordinates functions we obtain: 
Lemma 2. Write $\Theta_{p}(t)=\left(\Theta_{p}^{1}(t), \Theta_{p}^{2}(t), \ldots, \Theta_{p}^{n}(t)\right)$. Note that we can think of $X_{i}(x)$ as the $i$-th row of $\mathbb{A}(x)$. Similarly $D \Theta_{p}^{k}(0)$ is the $k$-column of $\mathbb{A}(p)$ so that

$$
D \Theta_{p}(0)=\mathbb{A}(p)
$$

For the second derivative we get

$$
\left\langle D^{2} \Theta_{p}^{k}(0) h, h\right\rangle=\left\langle\mathbb{A}^{t}(p) h, D\left(\mathbb{A}^{t}(p) h\right)_{k}\right\rangle
$$

for all vectors $h \in \mathbb{R}^{n}$.

In particular, the mapping $t \mapsto \Theta_{p}(t)$ is a diffeomorphism taking a neighborhood of 0 into a neighborhood of $p$.

A natural question is how $D_{\mathfrak{X}} u$ and $\left(D_{\mathfrak{X}}^{2} u\right)^{*}$ change if we change frames. The following lemma details how to change from the canonical frame $\left\{\frac{\partial}{\partial x_{1}}, \frac{\partial}{\partial x_{2}}, \ldots \frac{\partial}{\partial x_{n}}\right\}$ to the frame $\mathfrak{X}$ and follows easily from the definitions of $D_{\mathfrak{X}} u,\left(D_{\mathfrak{X}}^{2} u\right)^{*}$ and the chain rule. We denote the gradient relative to the canonical frame by $\nabla$.

Lemma 3. For smooth functions u we have

$$
D_{\mathfrak{X}} u=\mathbb{A} \cdot \nabla u,
$$

and for all $t \in \mathbb{R}^{n}$

$$
\left\langle\left(D_{\mathfrak{X}}^{2} u\right)^{*} \cdot t, t\right\rangle=\left\langle\mathbb{A} \cdot D^{2} u \cdot \mathbb{A}^{t} \cdot t, t\right\rangle+\sum_{k=1}^{n}\left\langle\mathbb{A}^{t} \cdot t, \nabla\left(\mathbb{A}^{t} \cdot t\right)_{k}\right\rangle \frac{\partial u}{\partial x_{k}} .
$$

The Taylor series gives the first version of the comparison principle for smooth functions.

Lemma 4. Let $u$ and $v$ be smooth functions such that $u-v$ has an interior local maximum at $p$. Then we have

$$
D_{\mathfrak{X}} u(p)=D_{\mathfrak{X}} v(p)
$$

and

$$
\left(D_{\mathfrak{X}}^{2} u(p)\right)^{*} \leq\left(D_{\mathfrak{X}}^{2} v(p)\right)^{*} .
$$

Let us consider some examples:

Example 1. The canonical frame

This is just $\left\{\frac{\partial}{\partial x_{1}}, \frac{\partial}{\partial x_{2}}, \ldots \frac{\partial}{\partial x_{n}}\right\}$. The first and second derivatives are just the usual ones and the exponential mapping is just addition

$$
\Theta_{p}(t)=p+t .
$$

Example 2. The Heisenberg group

We consider the Riemannian frame which is given by the left invariant vector fields $\left\{X_{1}, X_{2}, X_{3}\right\}$ in $\mathbb{R}^{3}$. For $p=(x, y, z)$ the matrix $\mathbb{A}$ is just

$$
\mathbb{A}(p)=\left(\begin{array}{ccc}
1 & 0 & -y / 2 \\
0 & 1 & x / 2 \\
0 & 0 & 1
\end{array}\right)
$$


A simple calculation shows that

$$
\left\langle\mathbb{A}^{t} \cdot t, D\left(\mathbb{A}^{t} \cdot t\right)_{k}\right\rangle=0
$$

not only for $k=1$ and $k=2$, but also for $k=3$. That is, although $\mathbb{A}$ is not constant, we have that Lemma 3 simplifies to

$$
\left\langle\left(D_{\mathfrak{X}}^{2} u\right)^{*} \cdot t, t\right\rangle=\left\langle\mathbb{A} \cdot D^{2} u \cdot \mathbb{A}^{t} \cdot t, t\right\rangle .
$$

The exponential mapping is just the group multiplication

$$
\Theta_{p}(t)=p \cdot \Theta_{0}(t)=\left(x+t_{1}, y+t_{2}, z+t_{3}+(1 / 2)\left(x t_{2}-y t_{1}\right)\right) .
$$

From Lemma (3) we see that the additional simplification of (2.3) occurs whenever $D^{2} \Theta_{p}^{k}(0)=0$. In particular this is true for all step 2 groups as it can be seen from the Campbell-Hausdorff formula.

\section{Example 3. The Engel group}

This is a step 3 group for which the analogue of (2.3) does not work. Denote by $p=(x, y, z, w)$ a point in $\mathbb{R}^{4}$. The Riemannian frame is given by the vector fields:

$$
\left\{\begin{array}{l}
X_{1}=\frac{\partial}{\partial x}-\frac{y}{2} \frac{\partial}{\partial z}+\left(\frac{-x y}{12}-\frac{z}{2}\right) \frac{\partial}{\partial w} \\
X_{2}=\frac{\partial}{\partial y}+\frac{x}{2} \frac{\partial}{\partial z}+\frac{x^{2}}{12} \frac{\partial}{\partial w} \\
X_{3}=\frac{\partial}{\partial z}+\frac{x}{2} \frac{\partial}{\partial w} \\
X_{4}=\frac{\partial}{\partial w}
\end{array}\right.
$$

So that the matrix $\mathbb{A}$ is

$$
\mathbb{A}=\left(\begin{array}{cccc}
1 & 0 & -\frac{y}{2} & \frac{-x y}{12}-\frac{z}{2} \\
0 & 1 & \frac{x}{2} & \frac{x^{2}}{12} \\
0 & 0 & 1 & \frac{x}{2} \\
0 & 0 & 0 & 1
\end{array}\right)
$$

Let $t=\left(t_{1}, t_{2}, t_{3}, t_{4}\right)$. Direct calculations shows that

$$
\begin{aligned}
& \left\langle\mathbb{A}^{t} \cdot t, D\left(\mathbb{A}^{t} \cdot t\right){ }_{1}\right\rangle=0 \\
& \left\langle\mathbb{A}^{t} \cdot t, D\left(\mathbb{A}^{t} \cdot t\right)_{2}\right\rangle=0 \\
& \left\langle\mathbb{A}^{t} \cdot t, D\left(\mathbb{A}^{t} \cdot t\right)_{3}\right\rangle=0
\end{aligned}
$$

but for $k=4$ we get

$$
\left\langle\mathbb{A}^{t} \cdot t, D\left(\mathbb{A}^{t} \cdot t\right)_{k}\right\rangle=-\frac{x}{6} t_{1} t_{2}+\frac{y}{6} t_{1}^{2} .
$$

Therefore, for a smooth function $u$ we have

$$
D_{\mathfrak{X}} u=\mathbb{A} \cdot D u,
$$

and for all $t \in \mathbb{R}^{n}$

$$
\left\langle\left(D_{\mathfrak{X}}^{2} u\right)^{*} \cdot t, t\right\rangle=\left\langle\mathbb{A} \cdot D^{2} u \cdot \mathbb{A}^{t} \cdot t, t\right\rangle+\left(-\frac{x}{6} t_{1} t_{2}+\frac{y}{6} t_{1}^{2}\right) \frac{\partial u}{\partial w} .
$$




\section{JETS}

To define second order superjets of an upper-semicontinuous function $u$, let us consider smooth functions $\varphi$ touching $u$ from above at a point $p$.

$$
\begin{array}{r}
K^{2,+}(u, p)=\left\{\varphi \in C^{2} \text { in a neighborhood of } p, \varphi(p)=u(p),\right. \\
\varphi(q) \geq u(q), q \neq p \text { in a neighborhood of } p\}
\end{array}
$$

Each function $\varphi \in K^{2,+}(u, p)$ determines a pair $(\eta, X)$ by

$$
\begin{aligned}
\eta & =\left(X_{1} \varphi(p), X_{2} \varphi(p), \ldots, X_{n} \varphi(p)\right) \\
A_{i j} & =\frac{1}{2}\left(X_{i}\left(X_{j}(\varphi)\right)(p)+X_{j}\left(X_{i}(\varphi)\right)(p)\right) .
\end{aligned}
$$

This representation clearly depends on the frame $\mathfrak{X}$. Using the Taylor theorem for $\varphi$ and the fact that $\varphi$ touches $u$ from above at $p$ we get

$$
u\left(\Theta_{p}(t)\right) \leq u(p)+\langle\eta, t\rangle+\frac{1}{2}\langle X t, t\rangle+o\left(|t|^{2}\right) .
$$

We may also consider $J_{\mathfrak{X}}^{2,+}(u, p)$ defined as the collections of pairs $(\eta, X)$ such that (3.2) holds. Using the identification given by (3.1) it is clear that

$$
K^{2,+}(u, p) \subset J_{\mathfrak{X}}^{2,+}(u, p) .
$$

In fact, we have equality. This is the analogue of the Crandall-Ishii Lemma of $[\mathrm{C}]$.

\section{Lemma 5.}

$$
K^{2,+}(u, p)=J_{\mathfrak{X}}^{2,+}(u, p) .
$$

Proof. Given a pair $(\eta, X) \in J_{\mathfrak{X}}^{2,+}(u, p)$ we must find a $C^{2}$ function $\varphi$ so that (3.1) holds. Given any pair $(\xi, Y)$ the version of the lemma for the canonical frame in $[\mathrm{C}]$ gives a $C^{2}$ function $\varphi$ touching $u$ from above at $p$ such that $D \varphi(p)=\xi$ and $D^{2} \varphi(p)=Y$. Using lemma 3 we get

$$
D_{\mathfrak{X}} \varphi(p)=\mathbb{A}(p) \cdot \xi
$$

and

$$
\left\langle\left(D_{\mathfrak{X}}^{2} \varphi\right)^{*} \cdot t, t\right\rangle=\left\langle\mathbb{A} \cdot Y \cdot \mathbb{A}^{t} \cdot t, t\right\rangle+\sum_{k=1}^{n}\left\langle\mathbb{A}^{t} \cdot t, D\left(\mathbb{A}^{t} \cdot t\right)_{k}\right\rangle \xi_{k} .
$$

Thus, it suffices to solve for $(\xi, Y)$ the equations

$$
\eta=\mathbb{A}(p) \cdot \xi
$$

and

$$
\langle X \cdot t, t\rangle=\left\langle\mathbb{A} \cdot Y \cdot \mathbb{A}^{t} \cdot t, t\right\rangle+\sum_{k=1}^{n}\left\langle\mathbb{A}^{t} \cdot t, D\left(\mathbb{A}^{t} \cdot t\right)_{k}\right\rangle \xi_{k}
$$


Theorem 1. The MAXIMUM PRINCIPLE FOR SEMICONTINUOUS FUNCTIONS Let $u$ be upper semi-continuous in a bounded domain $\Omega \subset \mathbb{R}^{n}$. Let $v$ be lower semi-continuous in $\Omega$. Suppose that for $x \in \partial \Omega$ we have

$$
\limsup _{y \rightarrow x} u(y) \leq \liminf _{y \rightarrow x} v(y),
$$

where both sides are not $+\infty$ or $-\infty$ simultaneously. If $u-v$ has a positive interior local maximum

$$
\sup _{\Omega}(u-v)>0
$$

then we have:

For $\tau>0$ we can find points $p_{\tau}, q_{\tau} \in \mathbb{R}^{n}$ such that

i)

$$
\lim _{\tau \rightarrow \infty} \tau \psi\left(p_{\tau}, q_{\tau}\right)=0
$$

where

$$
\psi(p, q)=|p-q|^{\alpha},
$$

for a fixed $\alpha \geq 2$.

ii) There exists a point $\hat{p} \in \Omega$ such that $p_{\tau} \rightarrow \hat{p}$ (and so does $q_{\tau}$ by (i)) and

$$
\sup _{\Omega}(u-v)=u(\hat{p})-v(\hat{p})>0,
$$

iii) there exist symmetric matrices $\mathcal{X}_{\tau}, \mathcal{Y}_{\tau}$ and vectors $\eta_{\tau}^{+}, \eta_{\tau}^{-}$so that

iv)

v)

$$
\left(\eta_{\tau}^{+}, \mathcal{X}_{\tau}\right) \in \bar{J}_{\mathfrak{X}}^{2,+}\left(u, p_{\tau}\right)
$$

$$
\left(\eta_{\tau}^{-}, \mathcal{Y}_{\tau}\right) \in \bar{J}_{\mathfrak{X}}^{2,-}\left(v, q_{\tau}\right),
$$

vi)

vi)

$$
\eta_{\tau}^{+}-\eta_{\tau}^{-}=o(1)
$$

and

$$
\mathcal{X}_{\tau} \leqq \mathcal{Y}_{\tau}+o(1)
$$

as $\tau \rightarrow \infty$.

Proof. The idea of the proof is to use the Euclidean theorem to get the jets and then twist them into position. As in the Euclidean case we get points $p_{\tau}$ and $q_{\tau}$ so that (i) and (ii) hold. We apply now the Euclidean maximum principle for semicontinuous functions of Crandall-Ishii-Lions [CIL]. There exist $n \times n$ symmetric matrices $X_{\tau}, Y_{\tau}$ so that

$$
\left(\tau D_{p}\left(\psi\left(p_{\tau}, q_{\tau}\right)\right), X_{\tau}\right) \in \bar{J}_{\text {eucl. }}^{2,+}\left(u, p_{\tau}\right)
$$

and

with the property

$$
\left(-\tau D_{q}\left(\psi\left(p_{\tau}, q_{\tau}\right)\right), Y_{\tau}\right) \in \bar{J}_{\text {eucl. }}^{2,-}\left(v, q_{\tau}\right)
$$

$$
\left\langle X_{\tau} \gamma, \gamma\right\rangle-\left\langle Y_{\tau} \chi, \chi\right\rangle \leqq\langle C \gamma \oplus \chi, \gamma \oplus \chi\rangle
$$


where the vectors $\gamma, \chi \in \mathbb{R}^{n}$, and

$$
C=\tau\left(A^{2}+A\right)
$$

and

$$
A=D_{p, q}^{2}\left(\psi\left(p_{\tau}, q_{\tau}\right)\right)
$$

are $2 n \times 2 n$ matrices.

Let us now twist the jets according to lemma 3. Call $\xi_{\tau}^{+}=\tau D_{p}\left(\psi\left(p_{\tau}, q_{\tau}\right)\right)$ and $\xi_{\tau}^{-}=-\tau D_{q}\left(\psi\left(p_{\tau}, q_{\tau}\right)\right)$. By our choice of $\psi$ we get $\xi_{\tau}^{+}=\xi_{\tau}^{-}$. Set

$$
\eta_{\tau}^{+}=\mathbb{A}\left(p_{\tau}\right) \cdot \xi_{\tau}^{+}
$$

and

$$
\eta_{\tau}^{-}=\mathbb{A}\left(q_{\tau}\right) \cdot \xi_{\tau}^{-}
$$

We see that

$$
\begin{aligned}
\left|\eta_{\tau}^{+}-\eta_{\tau}^{-}\right| & =\left|\mathbb{A}\left(p_{\tau}\right)-\mathbb{A}\left(q_{\tau}\right)\right|\left|\xi_{\tau}^{+}\right| \\
& \leq C \tau\left|p_{\tau}-q_{\tau}\right|\left|D_{p}\left(\psi\left(p_{\tau}, q_{\tau}\right)\right)\right| \\
& \leq C \tau \psi\left(p_{\tau}, q_{\tau}\right) \\
& =o(1),
\end{aligned}
$$

where we have used the fact that $|p-q|\left|D_{p} \psi(p, q)\right| \leq C \psi(p, q)$, property (i) and the smoothness, in the form of a Lipschitz condition, of $\mathbb{A}(p)$.

The second order parts of the jets are given by

$$
\left\langle\mathcal{X}_{\tau} \cdot t, t\right\rangle=\left\langle\mathbb{A}\left(p_{\tau}\right) X_{\tau} \mathbb{A}^{t}\left(p_{\tau}\right) \cdot t, t\right\rangle+\sum_{k=1, n}\left\langle\mathbb{A}^{t}\left(p_{\tau}\right) \cdot t, D\left(\mathbb{A}^{t}(p) \cdot t\right)_{k}\left[p_{\tau}\right]\right\rangle\left(\xi_{\tau}^{+}\right)_{k}
$$

and

$$
\left\langle\mathcal{Y}_{\tau} \cdot t, t\right\rangle=\left\langle\mathbb{A}\left(q_{\tau}\right) Y_{\tau} \mathbb{A}^{t}\left(q_{\tau}\right) \cdot t, t\right\rangle+\sum_{k=1, n}\left\langle\mathbb{A}^{t}\left(q_{\tau}\right) \cdot t, D\left(\mathbb{A}^{t}(p) \cdot t\right)_{k}\left[q_{\tau}\right]\right\rangle\left(\xi_{\tau}^{-}\right)_{k}
$$

In order to estimate their difference we write

$$
\begin{aligned}
\left\langle\mathcal{X}_{\tau} \cdot t, t\right\rangle-\left\langle\mathcal{Y}_{\tau} \cdot t, t\right\rangle= & \left\langle X_{\tau} \mathbb{A}^{t}\left(p_{\tau}\right) \cdot t, \mathbb{A}^{t}\left(p_{\tau}\right) \cdot t\right\rangle-\left\langle Y_{\tau} \mathbb{A}^{t}\left(q_{\tau}\right) \cdot t, \mathbb{A}^{t}\left(q_{\tau}\right) \cdot t\right\rangle \\
& +\sum_{k=1}^{n}\left\langle\mathbb{A}^{t}\left(p_{\tau}\right) \cdot t, D\left(\mathbb{A}^{t}(p) \cdot t\right)_{k}\left[p_{\tau}\right]\right\rangle\left(\xi_{\tau}^{+}\right)_{k} \\
& -\sum_{k=1}^{n}\left\langle\mathbb{A}^{t}\left(q_{\tau}\right) \cdot t, D\left(\mathbb{A}^{t}(p) \cdot t\right)_{k}\left[q_{\tau}\right]\right\rangle\left(\xi_{\tau}^{-}\right)_{k} .
\end{aligned}
$$

Using inequality 3.3 , we get

$$
\begin{aligned}
\left\langle\mathcal{X}_{\tau} \cdot t, t\right\rangle-\left\langle\mathcal{Y}_{\tau} \cdot t, t\right\rangle \leq & \left\langle C\left(\mathbb{A}\left(p_{\tau}\right) \cdot t \oplus \mathbb{A}\left(q_{\tau}\right) \cdot t\right), \mathbb{A}\left(p_{\tau}\right) \cdot t \oplus \mathbb{A}\left(q_{\tau}\right) \cdot t\right\rangle \\
+ & \tau\left[\sum_{k=1}^{n}\left\langle\mathbb{A}^{t}\left(p_{\tau}\right) \cdot t, D\left(\mathbb{A}^{t}(p) \cdot t\right)_{k}\left[p_{\tau}\right]\right\rangle \frac{\partial \psi}{\partial p_{k}}\left(p_{\tau}, q_{\tau}\right)\right] \\
& -\tau\left[\sum_{k=1}^{n}\left\langle\mathbb{A}^{t}\left(q_{\tau}\right) \cdot t, D\left(\mathbb{A}^{t}(p) \cdot t\right)_{k}\left[q_{\tau}\right]\right\rangle \frac{\partial \psi}{\partial p_{k}}\left(p_{\tau}, q_{\tau}\right)\right]
\end{aligned}
$$


To estimate the first term in the right hand side we note that symmetries of $\psi$ give a block structure to $D_{p, q}^{2} \psi$ so that we have

$$
\langle C(\gamma \oplus \delta), \gamma \oplus \delta) \leq C \tau|\gamma-\delta|^{2} .
$$

Replacing $\gamma$ by $\mathbb{A}\left(p_{\tau}\right) \cdot t$ and $\delta$ by $\mathbb{A}\left(q_{\tau}\right) \cdot t$, using the smoothness of $\mathbb{A}$, and property (i) we get that this first term is $o(1)$. The second and third term together are also $o(1)$ since their difference is estimated by a constant times $\tau\left|p_{\tau}-q_{\tau}\right|\left|D_{p} \psi\left(p_{\tau}, q_{\tau}\right)\right|$.

3.1. Fully Non-Linear Elliptic Equations. Consider a continuous function

$$
\begin{aligned}
F: & \mathbb{R}^{n} \times \mathbb{R} \times \mathbb{R}^{n} \times S\left(\mathbb{R}^{n}\right) \longrightarrow \mathbb{R} \\
& (x, z, \eta, \mathcal{X}) \longrightarrow F(x, z, \eta, \mathcal{X}) .
\end{aligned}
$$

We will always assume that $F$ is proper; that is, $F$ is increasing in $u$ and $F$ is decreasing in $\mathcal{X}$.

Definition 1. A lower semicontinuous function $v$ is a viscosity supersolution of the equation

$$
F\left(x, u(x), D_{\mathfrak{X}} u(x),\left(D_{\mathfrak{X}}^{2} u(x)\right)^{*}\right)=0
$$

if whenever $(\eta, \mathcal{Y}) \in J_{\mathfrak{X}}^{2,-}\left(v, x_{0}\right)$ we have

$$
F\left(x_{0}, v\left(x_{0}\right), \eta, \mathcal{Y}\right) \geq 0 \text {. }
$$

Equivalently, if $\varphi \in C^{2}$ touches $v$ from below at $x_{0}$, then we must have

$$
F\left(x_{0}, v\left(x_{0}\right), D_{\mathfrak{X}} \varphi\left(x_{0}\right),\left(D_{\mathfrak{X}}^{2} \varphi\left(x_{0}\right)\right)^{*}\right) \geq 0 .
$$

Definition 2. An upper semicontinuous function $u$ is a viscosity subsolution of the equation

$$
F\left(x, u(x), D_{\mathfrak{X}} u(x),\left(D_{\mathfrak{X}}^{2} u(x)\right)^{*}\right)=0
$$

if whenever $(\eta, \mathcal{X}) \in J_{\mathfrak{X}}^{2,+}\left(u, x_{0}\right)$ we have

$$
F\left(x_{0}, u\left(x_{0}\right), \eta, \mathcal{X}\right) \leq 0 .
$$

Equivalently, if $\varphi \in C^{2}$ touches $u$ from above at $x_{0}$, then we must have

$$
F\left(x_{0}, u\left(x_{0}\right), D_{\mathfrak{X}} \varphi\left(x_{0}\right),\left(D_{\mathfrak{X}}^{2} \varphi\left(x_{0}\right)\right)^{*}\right) \leqq 0 .
$$

Note that if $u$ is a viscosity subsolution and $(\eta, \mathcal{X}) \in \bar{J}_{\mathfrak{X}}^{2,+}\left(u, x_{0}\right)$ then, by the continuity of $F$, we still have

$$
F\left(x_{0}, u\left(x_{0}\right), \eta, \mathcal{X}\right) \leq 0 .
$$

A similar remark applies to viscosity supersolutions and the closure of second order subjets.

A viscosity solution is defined as being both a viscosity subsolution and a viscosity supersolution. Observe that since $F$ is proper, it follows easily that if $u$ is a smooth classical solution then $u$ is a viscosity solution. 


\section{Examples:}

- The Hörmander-Kohn operator relative to the frame $\mathfrak{X}$ :

$$
-\Delta_{\mathfrak{X}} u=-\left(\sum_{j=1}^{n} X_{j} X_{j} u\right)=0
$$

- The $\infty$-Laplace equation ([Bi]) relative to the frame $\mathfrak{X}$ :

$$
-\Delta_{\mathfrak{X}, \infty} u=-\left[\sum_{i, j=1}^{n}\left(X_{i} u\right)\left(X_{j} u\right) X_{i} X_{j} u\right]=-\left\langle\left(D_{\mathfrak{X}}^{2} u\right)^{*} D_{\mathfrak{X}} u, D_{\mathfrak{X}} u\right\rangle
$$

- The $p$-Laplace equation, $2 \leq p<\infty$, relative to the frame $\mathfrak{X}$ :

$$
\begin{aligned}
-\Delta_{\mathfrak{X}, p} u & =-\left[\left|D_{\mathfrak{X}} u\right|^{p-2} \Delta_{\mathfrak{X}} u+(p-2)\left|D_{\mathfrak{X}} u\right|^{p-4} \Delta_{\mathfrak{X}, \infty} u\right] \\
& =-\operatorname{div}\left(\left|D_{\mathfrak{X} u} u\right|^{p-2} D_{\mathfrak{X}} u\right)=0
\end{aligned}
$$

Strictly speaking we need $p \geq 2$ for the continuity of the corresponding $F$. In the Euclidean case it is possible to extend the definition to the full range $p>1$. This is a non-trivial matter not yet studied in the case of vector fields (to the best of our knowledge.) See [JLM] for the Euclidean case.

- The Monge-Ampère equation relative to the frame $\mathfrak{X}$ :

$$
-\operatorname{det}\left(D_{\mathfrak{X}}^{2} u\right)^{*}=f
$$

Here the corresponding $F(\mathcal{X})=-\operatorname{det} \mathcal{X}$ is only proper in the cone of positive semidefinite matrices.

Once we have the maximum principle (theorem 1) we get comparison theorems for viscosity solutions of many classes of fully nonlinear equations of the general form

$$
F\left(x, u(x), D_{\mathfrak{X}} u(x),\left(D_{\mathfrak{X}}^{2} u(x)\right)^{*}\right)=0
$$

where $F$ is continuous and proper as it is done in [CIL]. Here is an example:

Corollary 1. Suppose $F(x, z, \eta, \mathcal{X})$ satisfies

$$
\begin{aligned}
\sigma(r-s) & \leq F(x, r, \eta, \mathcal{X})-F(x, s, \eta, \mathcal{X}) \\
|F(x, r, \eta, \mathcal{X})-F(y, r, \eta, \mathcal{X})| & \leq \omega_{1}(|x-y|) \\
|F(x, r, \eta, \mathcal{X})-F(x, r, \eta, \mathcal{Y})| & \leq \omega_{2}(|\mathcal{X}-\mathcal{Y}|) \text { an } \\
|F(x, r, \eta, \mathcal{X})-F(x, r, \xi, \mathcal{X})| & \leq \omega_{3}(|\eta-\xi|)
\end{aligned}
$$

where the constant $\sigma>0$ and the functions $\omega_{i}:[0, \infty) \mapsto[0, \infty)$ satisfy $\omega_{i}\left(0^{+}\right)=0$ for $i=1,2,3$. Let $u$ be an upper-continuous viscosity solution and $v$ a lower semi-continuous viscosity supersolution to

$$
F\left(x, u(x), D_{\mathfrak{X}} u(x),\left(D_{\mathfrak{X}}^{2} u(x)\right)^{*}\right)=0
$$

in a domain $\Omega$ so that for all $p \in \partial \Omega$ we have

$$
\limsup _{q \in \Omega, q \rightarrow p} u(q) \leq \liminf _{q \in \Omega, q \rightarrow p} v(q)
$$


and both sides are not $\infty$ or $-\infty$ simultaneously. Then

$$
u(p) \leq v(p)
$$

for all $p \in \Omega$.

\section{REFERENCES}

[Bi] Bieske, T., On $\infty$-harmonic functions on the Heisenberg group, Comm. in PDE 27 (2002) no. $3 \& 4,727-761,2002$.

[C] Crandall, M., Viscosity Solutions: A Primer, Lecture Notes in Mathematics 1660, Springer-Verlag, 1997.

[CIL] Crandall, M., Ishii, H. and Lions, P. L., User's Guide to Viscosity Solutions of Second Order Partial Differential Equations, Bull. of Amer. Math. Soc., 27, No. 1, July 1992, pp. 1-67.

[J] Jensen, R., The maximum principle for viscosity solutions of fully nonlinear second order partial differential equations, Arch. Rational Mech. Anal. 101 (1988), no. $1,1-27$

[JLM] Juutinen, P., Lindqvist, P., and Manfredi, J., On the equivalence of viscosity solutions and weak solutions for a quasi-linear equation, SIAM J. of Math. Analysis 33 no. 3 (2001), 699-717.

[NSW] Nagel, A., Stein, E. and Wainger, S., Balls and metrics defined by vector fields I: Basic properties, Acta Math. 155, 1985, pp. 103-147.

[W] Wang, C., The Comparison Principle for Fully Non-Linear Equations on Carnot groups, preprint.

Department of Mathematics, University of Pittsburgh, Pittsburgh, PA 15260, USA

E-mail address: beatrous@pitt.edu

Department of Mathematics, University of South Florida, Tampa, FL 33620

E-mail address: tbieske@math.usf.edu

Department of Mathematics, University of Pittsburgh, Pittsburgh, PA 15260, USA

E-mail address: manfredi@pitt.edu 